\title{
Propuesta de ecuaciones para predecir la composición corporal de ratas machos wistar
}

\author{
Proposed equations for predicting body composition of male wistar rats
}

\author{
Marco Antonio Cossio-Bolaños ${ }^{1 a, 2}$, Rossana Gómez ${ }^{3 a}$, Julio Rojas ${ }^{4 b}$, Haroldo Flores ${ }^{4 c}$ \\ ${ }^{1}$ Instituto del Deporte Universitario, Universidad Nacional de San Agustín. Arequipa, Perú. \\ ${ }^{2}$ Departamento de Ciência do desporto, Faculdade de Educação física, Universidade Estadual de Campinas. São Paulo, Brasil. \\ ${ }^{3}$ Escuela Internacional de Educación Física y Deportes. La Habana, Cuba. \\ ${ }^{4}$ Departamento de Farmacologia, Faculdade de Ciências Médicas, Universidade Estadual de Campinas. São Paulo, Brasil.

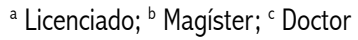

\begin{abstract}
Resumen
Introducción: La evaluación de la composición corporal es importante, porque permite conocer el fraccionamiento del peso corporal en peso muscular, peso graso, peso residual y peso óseo, tanto en humanos, como en animales. Objetivos: Validar ecuaciones somáticas para predecir la composición corporal de un modelo tri-compartimental (peso graso, peso libre de grasa y peso residual) de ratas machos wistar. Diseño: Estudio de tipo descriptivo de corte transversal. Institución: Facultad de Biología de la universidad estatal UNICAMP, Sao Paulo, Brasil. Material biológico: Ratas machos wistar. Métodos: Se estudió a 10 ratas machos wistar, con un promedio de edad de ( $X=98,00 \pm 10,40$ días), se les evaluó el peso corporal (g). Enseguida fueron sacrificados y se procedió al fraccionamiento del peso graso (piel), peso libre de grasa (músculo y hueso) y peso residual (PR) (g). Los resultados fueron analizados por estadística descriptiva de media aritmética $(X)$, desviación estándar (DE) y correlación producto momento de Pearson (r). Para predecir los componentes, se aplicó regresiones estadísticas simples y múltiples, a partir del peso corporal (g) y la edad (días) como variables independientes. Por otro lado, para verificar la concordancia entre el método directo de disección in vitro con las ecuaciones de regresión, se utilizó el plotaje de Bland y Altman. Principales medidas de resultados: Validación de ecuaciones somáticas para predecir la composición corporal de ratas. Resultados: Se verificó altos coeficientes de correlación ( $r$ ) con el peso corporal y edad, los cuales dieron origen a ecuaciones que permitieron predecir el peso graso ( $P G=-31,6+\left(0,361^{*} \mathrm{PT}\right)-\left(0,345^{*}\right.$ edad $)$ $(R 2=0,73)$ y peso libre de grasa $\left(P L G=19,9+(0,453 * P T)+\left(0,114^{*}\right.\right.$ edad $)$ $(R 2=0,94)$. Sin embargo, el peso residual $(P R)$ fue obtenido por medio de una deducción matemática ( $P R=$ peso total-( $P L G+P G))$. Así mismo, el plotaje de Bland y Altman permitió determinar alta concordancia entre ambos procedimientos. Conclusión: Las ecuaciones de regresión como método doblemente indirecto (modelo tri-compartimental) permiten predecir la composición corporal de ratas machos wistar en una fase etárea de 84 a 112 días de edad.
\end{abstract}

Palabras clave: Composición corporal; peso corporal; ratas wistar.

\begin{abstract}
Introduction: Assessment of body composition is important as it allows splitting body weight in muscle weight, fat weight, bone weight and residual weight, both in humans and animals. Objectives: To validate somatic equations to predict a tricompartment model of body composition (fat weight, fat free weight and residual weight) in male Wistar rats. Design: Descriptive transversal type study. Setting: Faculty of Biology, State University UNICAMP, Sao Paulo, Brazil. Biological material: Male Wistar rats. Methods: The study evaluated body weight $(\mathrm{g})$ in 10 average age $(X=98.00 \pm 10.40$ days) male Wistar rats. They were sacrificed and splitting of fat weight (skin), fat free weight (muscle and bone) and residual weight (PR) ( $g$ ) was done. Results were analyzed by arithmetic mean (X), standard deviation (SD) and Pearson product moment correlations $(r)$ descriptive statistics. To predict components single and multiple regression statistics were applied, with weight body ( $\mathrm{g}$ ) and age (days) as independent variables. On the other hand, to verify agreement between in vitro method of dissection and regression equations Bland and Altman's plotting were used. Main outcome measures: Somatic equations validation to predict rat's corporal composition. Results: We observed high correlation coefficients $(r)$ with body weight and age, which led to equations to allow prediction of the fat weight $\left(\mathrm{PG}=-31.6+(0.361 * \mathrm{PT})-\left(0.345^{*}\right.\right.$ age $)(\mathrm{R} 2=0.73)$ and fat free weight $(P L G=19.9+(0.453 * P T)+(0.114 *$ age $)(R 2=0.94)$. However, residual weight $(P R)$ was obtained by mathematical deduction $(P R=$ total weight $-(P L G+P G))$. Additionally, the Bland and Altman's plotting allowed determining high concordance between the two procedures. Conclusion: Regression equations as doubly indirect method (three-compartment model) allow predicting 84 to 112 day-old male Wistar rats' body composition.
\end{abstract}

Key words: Body composition; body weight; rats, wistar.

\section{INTRODUCCIÓN}

La evaluación de la composición corporal es extremadamente útil en estudios de la fisiología, tanto en humanos como en animales ${ }^{(1)}$, dado que numerosos estudios en animales pequeños requieren las medidas exactas de la composición del cuerpo en forma bruta ${ }^{(2)}$. La composición corporal evalúa la cantidad y la proporción de los principales componentes estructurales del organismo a través del fraccionamiento del peso corporal (3), basándose en la separación del peso en diferentes compartimientos, cuya suma es igual al peso corporal total ${ }^{(4)}$. De esa forma, el análisis del fraccionamiento en modelos de dos, tres, cuatro y cinco componentes se puede determinar por varios métodos, destacando Dahms, Glass (5) que la composición corporal puede ser evaluada por métodos directos e indirectos, es decir, por medio de la disección de la carcasa y análisis químico ${ }^{(6)}$. Sin embargo, esos procedimientos implican mucho tiempo y sus procedimientos son tediosos, permitiendo una sola evaluación y requiere sacrificio de los animales, no posibilitando estudios longitudinales, así como tratamientos de dietas e intervenciones ${ }^{(7)}$. Por ello, es importante estudiar los cambios de la composición del peso corporal de los animales durante la etapa del crecimiento ${ }^{(8)}$.

Desde esa perspectiva, varios estudios en ratas fueron desarrollados utilizando métodos no-invasivos, como el método de absormetría de doble energía DEXA para la evaluación de la composición corporal ${ }^{(7,9,10)}$ y de rayos $\mathrm{X}$, resonancia 
magnética de imagen técnica ${ }^{(11,12)}$. Sin embargo, el elevado costo y la accesibilidad al instrumental imposibilitan su uso y aplicación. Por lo tanto, el objetivo del presente estudio es validar ecuaciones para predecir la composición corporal de un modelo tri-compartimental (peso graso (PG), peso libre de grasa y peso residual) de ratas machos wistar.

\section{MÉTODOS}

El estudio es de tipo descriptivo de corte transversal ${ }^{(13)}$, estudiando 10 ratas machos sedentarios de raza wistar, con una edad media de $(X=98,00 \pm 10,40$ días $)$, provenientes del Bioterio del Departamento de Farmacología de la Universidad Estatal de Campinas (UNICAMP), Sao Paulo, Brasil. Los animales fueron alojados con vida durante el experimento en cajas colectivas con cinco animales en cada una, en ciclos de claro/oscuro (12/12 h), recibiendo ración patrón (Labina, Purina) y agua ad libitum. Se obtuvo la respectiva aprobación del Comité de Ética de la Facultad de Biología de la UNICAMP.

Los animales fueron pesados antes de ser sacrificados. Se consideró dos evaluaciones, como mínimo, de las cuatro evaluaciones realizadas, manteniendo siempre los cuidados necesarios de las interferencias de objetos extraños durante el proceso de medición. Las evaluaciones presentaron un error técnico de medida (ETM) de $(<0,70 \%)$ y un coeficiente de reproductibilidad de $(r=0,99)$.

Para el peso corporal (g), se utilizó una balanza analítica de marca Scaltec modelo SAC-62, con una precisión de $10^{-4} \mathrm{~g}$. El procedimiento consistió en colocar los animales en un frasco leve sobre la balanza, con el objetivo de evaluar el peso corporal sin que el animal se moviera constantemente.

Para la realización del fraccionamiento en compartimientos corporales, los animales fueron sacrificados por decapitación. Luego, se extrajo la sangre, los líquidos del organismo, vísceras y grasa interna de cada animal. Estos elementos constituyeron el peso residual (PR), como un primer componente. A continuación, se utilizó tijeras quirúrgicas para diseccionar el tejido adiposo (piel), con- juntamente con la grasa externa que se encuentra adherida a la masa muscular en lugares específicos, como el pescuezo, el abdomen, muslos y pelvis. Esta disección dio origen a un segundo componente, la masa grasa (MG).

El tercer componente estuvo constituido por la carcasa (músculo y hueso), el cual estuvo representado por el peso libre de grasa (PLG). En consecuencia, la sumatoria de los tres componentes dio como resultado el peso total (PT) del animal: $\mathrm{PT}=(\mathrm{PR}+\mathrm{PG}+\mathrm{PLG})$. Sin embargo, para efectos de realizar los cálculos del peso residual, sugerimos la siguiente deducción: $\mathrm{PR}=\mathrm{PT}-(\mathrm{PLG}+\mathrm{PG})$.

Para la validación de las ecuaciones, se utilizó el método directo (disección) in vitro, el cual es considerado como un criterio externo. A partir de dichos valores, se buscó las correlaciones y predicciones, utilizando como variables independientes el peso corporal y la edad. A este respecto, podemos señalar que la validez se caracteriza por considerar tres aspectos relevantes: validez de contenido, criterio y constructo.

En consecuencia, para el presente estudio se optó por la validez de criterio, utilizando como procedimientos la regresión simple y múltiple, puesto que dichos valores pueden ser interpretados en porcentaje de predicción, expresados en $\mathrm{R}^{2}$, tal y como fue utilizado en el Estudio de Cadáveres de Bruselas (Brussels Cadaver Study) ${ }^{(14)}$, en humanos.

Los resultados del presente estudio fueron analizados a través de la estadística descriptiva de la media $(\mathrm{X})$, desviación estándar (DE), coeficiente de variación (CV) y coeficiente de correlación de Pearson (r).

Para predecir los valores del tejido adiposo (peso graso) y la masa libre de

Tabla 1. Caracterización de la población estudiada $(n=10)$.

\begin{tabular}{lcccccc}
\hline & $X$ & DE & CV & EEE & Mínimo & Máximo \\
\hline Edad (días) & 98,0 & 10,40 & 10,61 & 3,30 & 84,00 & 112,00 \\
Masa corporal (g) & 408,6 & 34,37 & 8,41 & 10,90 & 345,00 & 465,50 \\
\hline
\end{tabular}

X: media aritmética; DE: desviación estándar; EEE: error estándar de estimación. gordura (MLG), se realizó regresiones estadísticas simples y múltiples a partir del peso corporal (g) y la edad en días, respectivamente.

Finalmente, para analizar la concordancia entre el método in vitro y las ecuaciones de predicción, se utilizó la prueba $t$ para las diferencias entre los métodos, así como el coeficiente intraclase de Spearman (CS) y la concordancia con el enfoque de componentes de la varianza, por medio del método de Bland y Altman ${ }^{(15)}$.

\section{RESULTADOS}

La tabla 1 muestra las características de la población estudiada, teniendo como variables de medición la edad (días) y la masa corporal (g). Esos valores estuvieron representados por la estadística descriptiva de media aritmética $(\mathrm{X})$, desviación estándar (DE), coeficiente de variación $(\mathrm{CV})$ y valores mínimo y máximo. Todas las variables evaluadas mostraron aceptables niveles de coeficiente de variación, siendo considerados por lo tanto homogéneos: edad $(10,61 \%)$ y peso $(8,41 \%)$.

El fraccionamiento de la composición corporal en tres componentes corporales está descrito en la tabla 2. Esos valores son producto de la disección de las ratas machos wistar in vitro, donde se observa los valores medios $(\mathrm{X})$, desviación estándar (DE), coeficiente de variación (CV) y error estándar de estimación (EEE) del peso graso $(\mathrm{g})$, peso libre de grasa $(\mathrm{g})$ y peso residual $(\mathrm{g})$, respectivamente. Estos valores fueron inferiores a $13,51 \%$, porcentaje considerado como homogéneo, puesto que no superó el $33 \%$.

En la tabla 3, se puede observar la relación entre los indicadores somáticos o medidas murinométricas de la masa 
Tabla 2. Valores promedios $(X)$ y desviación estándar (DE) de la composición corporal (modelo tri-compartimental) obtenidos a través del método referencia directo (disección in vitro).

\begin{tabular}{lcccc}
\hline & $X$ & DE & CV & EEE \\
\hline Peso libre de grasa $(\mathrm{g})$ & 216,3 & 16,94 & 7,83 & 5,36 \\
Peso de grasa $(\mathrm{g})$ & 82,10 & 11,10 & 13,51 & 3,51 \\
Peso residual $(\mathrm{g})$ & 110,10 & 8,970 & 8,14 & 2,84 \\
Peso total $(\mathrm{g})$ & 408,60 & 34,40 & 8,41 & 10,96 \\
\hline
\end{tabular}

$X$ : media aritmética; $D E$ : desviación estándar; $C V$ : coeficiente de variación; $E E E$ : error estándar de estimación.

Tabla 3. Correlación entre variables somáticas y componentes corporales (disección in vitro).

\begin{tabular}{lcccc}
\hline & PLG & PG & PR & PT \\
\hline Peso total (g) & 0,975 & 0,867 & 0,919 & --- \\
Edad (días) & 0,785 & 0,544 & 0,821 & 0,776 \\
\hline
\end{tabular}

PLG: peso libre de grasa; PG: peso graso; PR: peso residual; PT: peso total.

corporal (g) y la edad (días) con los componentes corporales disecados in vitro, de la masa libre de grasa (MLG), masa de grasa $(M G)$, masa residual (MR) y masa total. Las correlaciones para la masa libre de grasa oscilaron entre 0,98 y 0,79 , para la masa de grasa entre 0,87 y 0,54 , para la masa residual 0,92 y 0,82 y para la masa total con la edad fue 0,78 . Por lo tanto, esos valores mostraron fuertes correlaciones, las cuales fueron aprovechadas en el presente estudio para predecir la masa de grasa $(M G)$ y la masa libre de grasa (MLG), respectivamente.

Las variables murinométricas usadas para predecir el peso libre de grasa (PLG) y el peso de la grasa PG) se encuentran en la tabla 4. Los resultados de las regre-

siones estadísticas de los cuatro modelos matemáticos mostraron coeficientes de predicción altos, siendo de $98 \%$ para la predicción de PLG, tanto a partir del peso corporal como del peso corporal más la edad. Sin embargo, para predecir la MG los valores predictivos fueron inferiores, siendo de $72 \%$ la predicción a partir del peso corporal y de $73 \%$ a partir del peso corporal más la edad, respectivamente.

Estos resultados demuestran que, a partir de los indicadores somáticos, como es el caso del peso corporal de ratas, se puede predecir el PR, PLG y MG in vivo (ver figuras 1 y 2). En consecuencia, es posible realizar evaluaciones de la composición corporal antes, durante y después de tratamientos e intervenciones

Tabla 4. Ecuaciones para predecir el peso libre de grasa (PLG) y peso de grasa (PG) de ratas machos wistar.

\begin{tabular}{clcccc}
\hline $\mathrm{N}^{\circ}$ & Ecuación & $\mathrm{R}$ & $\mathrm{R}^{2}$ & $\mathrm{EEE}$ & $p$ \\
\hline 1 & $\mathrm{PLG}=20,1+\left(0,480^{*} \mathrm{PT}\right)$ & 0,975 & 0,944 & 4,012 & $<0,001$ \\
2 & $\mathrm{PLG}=19,9+\left(0,453^{*} \mathrm{PT}\right)+\left(0,114^{*} \mathrm{edad}\right)$ & 0,976 & 0,938 & 4,204 & $<0,001$ \\
3 & $\mathrm{PG}=-32,2+\left(0,280^{*} \mathrm{PT}\right)$ & 0,867 & 0,721 & 5,866 & $<0,001$ \\
4 & $\mathrm{PG}=-31,6+\left(0,361^{*} \mathrm{PT}\right)-\left(0,345^{*}\right.$ edad $)$ & 0,891 & 0,734 & 5,718 & $<0,001$ \\
\hline
\end{tabular}

PLG: peso libre de grasa; PG: peso graso; PR: peso residual; PT: peso total. en investigaciones experimentales y longitudinales en grupos específicos de ratas machos wistar en proceso de crecimiento somático.

La tabla 5 muestra los valores de concordancia de las ecuaciones obtenidas a través de regresiones estadísticas, para lo cual se consideró como método especifico la disección in vitro, a partir del cual se buscó la concordancia con cada uno de los cuatro modelos matemáticos.

$\mathrm{Al}$ respecto, cabe resaltar que existen varias técnicas que permiten evaluar la concordancia entre metodologías, considerando en el presente estudio la prueba t de Student, el coeficiente de correlación de Spearman, los valores medios de las diferencias entre los métodos y el análisis con el enfoque de los componentes de la varianza, por medio del método de plotaje de Bland y Altman (15).

Los resultados mostraron que, inicialmente, cuando se aplicó la t de Student para comparar el método especifico de disección in vivo con cada ecuación, no difirió significativamente con grupo alguno $(p>0,011)$. De la misma forma, cuando se aplicó el coeficiente intraclase de Spearman, los resultados mostraron valores próximos a 1 , siendo la correlación más fuerte para las ecuaciones del peso libre de grasa $(\mathrm{PLG})(\mathrm{S}=0,97-1,00)$ y relativamente inferiores para el peso de grasa $(P G)(S=0,88-0,94)$. Dichos resultados sugieren que tanto por medio de las ecuaciones y/o el método directo de disección in vitro se obtiene los mismos resultados. Sin embargo, cuando se aplicó el criterio de las diferencias de las medias, los resultados mostraron valores superiores a 3,71 , ya que los valores mínimos de diferencias entre las medias de dos comparaciones deben ser $\leq 1,4$.

Finalmente, cuando se analizó mediante el último criterio, los resultados mostraron que la totalidad de los datos se encontraban dentro de los intervalos, mostrando una concordancia alta $(\mathrm{C}=15,12$ y 14,83$)$ para el peso libre de gordura (PLG) y $(\mathrm{C}=20,14$ y 22,09) para la masa de grasa $(\mathrm{MG})$, respectivamente. Los resultados pueden ser observados en la tabla 5 y en las figuras 3 y 4 , donde el plotaje de Bland y Altman muestra mayor concentración de los datos. 


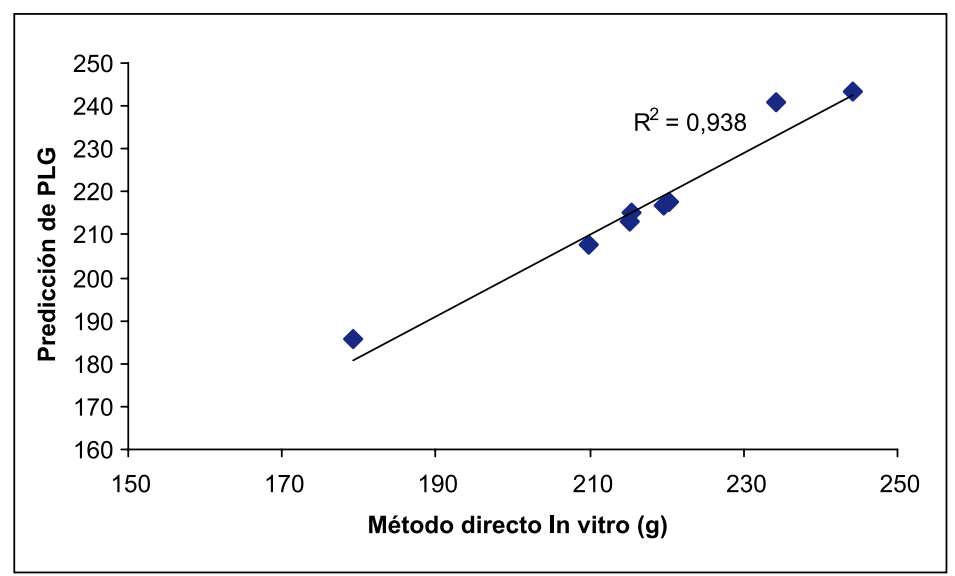

Figura 1. Relación entre los valores predichos y la técnica de disección in vitro de masa libre de grasa (MLG).

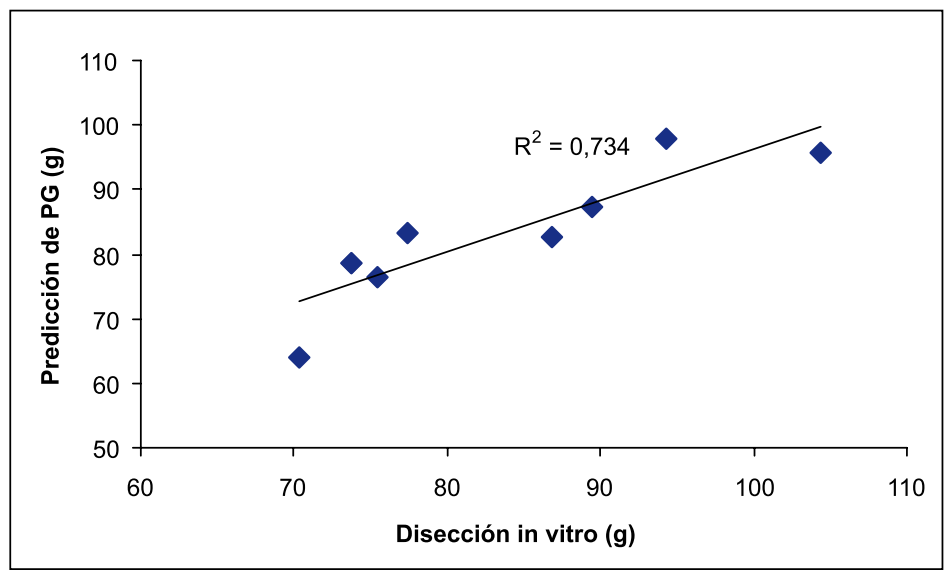

Figura 2. Relación entre los valores predichos y la técnica de disección in vitro de masa de grasa (PG).

\section{DISCUSIÓN}

Los resultados del presente estudio muestran que el peso corporal y la edad son buenos indicadores para predecir la composición corporal (modelo tri- compartimental) en peso libre de grasa (PLG), peso graso (PG) y peso residual (PR) de ratas machos wistar. Dado que para validar dichas ecuaciones se utilizó como método de criterio la disección completa de ratas in vitro, procedimiento conocido en los humanos como Estudio de Cadáveres de Bruselas ${ }^{(14)}$, pues en la evaluación de la composición corporal en humanos es ampliamente conocido que su medición puede ser efectuada a través de métodos directos, indirectos y doblemente indirectos ${ }^{(16-18)}$, siendo considerado el método directo como el de mayor precisión y exactitud ${ }^{(14,19)}$. Sin embargo, en animales, el análisis químico de la carcasa siempre fue considerado como el método estándar de oro ${ }^{(20-22)}$, ya que presenta exactitud y precisión en sus mediciones ${ }^{(21)}$.

Desde esa perspectiva, cabe resaltar que las ecuaciones obtenidas en el presente estudio muestran valores de explicación de $\left(\mathrm{R}^{2}=0,94\right)$ para el peso libre de grasa (PLG) y de $\left(\mathrm{R}^{2}=0,72-0,73\right)$ para el peso graso $(\mathrm{PG})$, siendo valores aceptables, ya que explican su predicción en $94 \%$ y $72-73 \%$, respectivamente. Estos valores son informados por otros estudios $\left(R^{2}=0,52-0,94\right)$ en ecuaciones propuestas para humanos ${ }^{(17,23)}$, teniendo como criterio de validación el pesaje hidrostático. A su vez, cabe resaltar que el error técnico de medida (ETM) del peso corporal mostró valores inferiores al 0,70\% y una capacidad de reproductibilidad de $\mathrm{r}=0,99$, por lo tanto, aplicables a ratas machos wistar comprendidos en edades de 84 a 112 días, respectivamente.

En ese sentido, en relación a estudios que impliquen el uso de animales pequeños para evaluar la composición corporal, Rose y col ${ }^{(24)}$ y Feely y col (25), consideran que existen fuertes correlaciones entre el método químico y la absorciometría de doble energía de rayos-X (DEXA), destacando algunas investigaciones valores aceptables de

Tabla 5. Valores de concordancia entre o método in vitro de modelos matemáticos de regresión estadística para predecir el peso libre de grasa y peso de grasa de ratas.

\begin{tabular}{|c|c|c|c|c|c|c|c|}
\hline \multicolumn{2}{|c|}{ Componentes } & \multirow{2}{*}{$\begin{array}{c}T \\
0,0159(n) \\
0,0238(n)\end{array}$} & \multirow{2}{*}{$\begin{array}{c}\text { Spearman } \\
0,97 \\
1,00\end{array}$} & \multirow{2}{*}{$\begin{array}{c}\begin{array}{c}\text { Media de las } \\
\text { diferencias }\end{array} \\
0,120 \\
0,178\end{array}$} & \multirow{2}{*}{$\begin{array}{c}\text { DE de las } \\
\text { diferencias }\end{array}$} & \multirow{2}{*}{$\begin{array}{c}\begin{array}{c}\text { Intervalo de } \\
\text { concordancia }\end{array} \\
-7,44 \text { a } 7,68 \\
-7,24 \text { a } 7,59\end{array}$} & \multirow{2}{*}{$\begin{array}{c}\text { Concordancia } \\
15,12 \\
14,83\end{array}$} \\
\hline PLG & $\begin{array}{l}\text { In vitro - PT } \\
\text { In vitro- } \mathrm{PT}+\text { edad }\end{array}$ & & & & & & \\
\hline PG & $\begin{array}{l}\text { In vitro - } \mathrm{PT} \\
\text { In vitro - } \mathrm{PT}+\text { edad }\end{array}$ & $\begin{array}{l}0,0146(n) \\
0,0105(n)\end{array}$ & $\begin{array}{l}0,94 \\
0,88\end{array}$ & $\begin{array}{l}-0,070 \\
-0,049\end{array}$ & $\begin{array}{l}5,530 \\
5,040\end{array}$ & $\begin{array}{c}-11,1 \text { a } 10,99 \\
-10,00 \text { a } 10,14\end{array}$ & $\begin{array}{l}22,09 \\
20,14\end{array}$ \\
\hline
\end{tabular}

DE: desviación estándar; PLG: peso libre de grasa; PG: peso graso; PT: peso total; n: no significativo. 


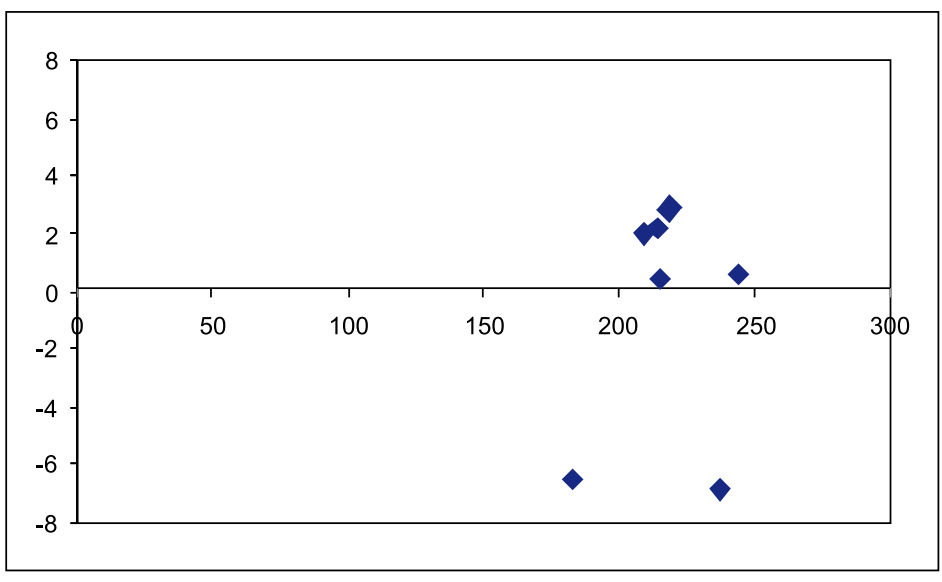

Figura 3. Valoración de la concordancia entre la técnica de disección in vitro y los valores predichos (peso+edad) del peso libre de grasa (PLG), a través del plotaje de Bland y Altman.

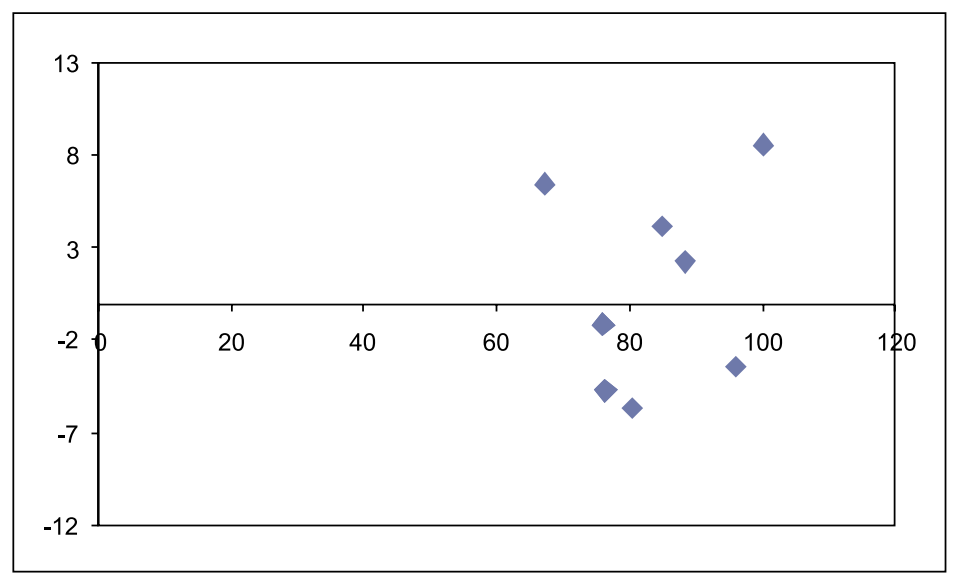

Figura 4. Valoración de la concordancia entre la técnica de disección in vitro y los valores predichos (peso+edad) de peso de grasa (PG), a través del plotaje de Bland y Altman.

reproductibilidad en ratas ${ }^{(24,26)}$ y gatos $(27,28)$. A pesar que el análisis químico de la carcasa es considerada como método oro (padrón) para validar la absorciometria DEXA ${ }^{(22)}$ y analizar la composición corporal de roedores, cabe resaltar que muchos laboratorios carecen de aparatos sofisticados y necesitan de técnicas no invasivas para predecir la composición corporal de ratas, como las ecuaciones propuestas en el presente estudio. De esa forma, cuando se pretende desarrollar estudios experimentales y longitudinales es posible el análisis del comportamiento de componentes corporales de peso libre de grasa (PLG), peso graso (PG) y peso residual (PR), antes, durante y después de los tratamientos y/o intervenciones, sin tener que sacrificar a los animales a expensas del análisis de la carcasa ${ }^{(2)}$.

Por lo tanto, las ecuaciones propuestas en el presente estudio son una herramienta fundamental para el diagnóstico de la composición corporal de ratas, aunque es necesario desarrollar más estudios que permitan el uso de otros indicadores somáticos.

Por los resultados obtenidos en el presente estudio, se concluye que las ecuaciones para el peso libre de gordura (PLG) y peso graso (PG) muestran altos valores de predicción (72 a 94\%) y correlación alta con el método directo de disección in vitro que fue usado como criterio de validación, siendo sugerido su empleo para ratas machos wistar comprendidos entre 84 a 112 días, respectivamente.

\section{REFERENCIAS BIBLIOGRÁFICAS}

1. Rutter K, Hennoste I, Ward LC, Cornish BH, Thomas BJ. Bioelectrical impedance analysis for the estimation of body composition in rats. Lab Anim. 1998;32:65-71.

2. Jebb SA, Garland SW, Jennings G, Elia M. Dualenergy $X$-ray absorptiometry for the measurement of gross body composition in rats. $\mathrm{Br} J$ Nutr. 1996;75:803-9.

3. Malina RM, Bouchard C. Growth, maturation and physical activity. Champaign: Human Kinetics; 1991.

4. Mcardle K. Fisiología del ejercicio: Energía, nutrición, y desempeño motor. 4ta ed. Rio de Janeiro: Editora Guanabara koogan; 1998.

5. Dahms W, Glass A. Correlation of percent body fat with body specific gravity in rats. J Nutr. 1982;112:398-400.

6. Reid JT (ed). Body Composition in Animals and Man. Washington, DC: National Academy of Science; 1969.

7. Lukaski HC, Hall CB, Marchello MJ, Siders WA. Validation of dual X-ray absorptiometry for bodycomposition assessment of rats exposed to dietary stressors. Nutrition. 2001;17:607-13.

8. Johnson MS, Nagy T. Animal body composition methods. En: Heymsfield SB, Lohman TG, Wang Z, Going S. Human Body composition. 2da ed. Champaign: Human Kinetics; 2005. p. 141-50.

9. Ammann P, Rizzoli R, Slosman D, Bonjour JP. Sequential and precise in vivo measurement of bone mineral density in rats using dualenergy x-ray absorptiometry. J Bone Miner Res. 1992;7:311-6.

10. Grier SJ, Turner AS, Alvis MR. The use of dualenergy $X$-ray absorptiometry in animals. Investig Radiol. 1996;31:50-62.

11.Sharp JC, Copps JC, Liu Q, Ryner LN, Sebastian $\mathrm{RA}$, Zeng GQ, et al. Analysis of ovariectomy and estrogen effects on body composition in rats by $X$-ray and magnetic resonance imaging techniques. J Bone Miner Res. 2000;15:13846.

12. Tang H, Wu EX, Gallagher D, Vasselli JR, Boozer CN. An in vivo validation study of body composition in rats using high resolution MRI. Proc Intl Soc Mag Reson. 2001;9:708.

13. Thomas J, Nelson J. Research methods in physical activity. Champaign: Human Kinetics; 1996.

14. Clarys JP, Martin AD, Drinkwater D. Gross tissue weights in the human body cadaver dissection. Human Biol. 1984;56:459-73.

15. Bland JM, Altman DG. Statistical methods for assessing agreement between two methods of clinical measurement. Lancet. 1986;1:307-10. 
16. Heymsfield SB, Wang Z, Baumgartner RN, Ross R. Human body composition: advances in models and methods. Annu Rev Nutr. 1997;17:527-58.

17. Peterson MJ, Czerwinski SA, Siervogel RM. Development and validation of skinfold-thickness prediction equations with a 4-compartment model. Am J Clin Nutr. 2003;77:1186-91.

18. Heyward VH, Wagner DR. Applied body composition. (2nd ed.). Champaign, IL: Human Kinetics; 2004.

19. Martin AD, Drinkwater DT, Clarys JP, Ross WD. Prediction of body fat skinfold calipers. Assumptions and cadaver evidence. Int J Obes. 1986;7:17-25.

20. Folch J, Lees M, Stanley GHS. A simple method for the isolation and purification of total lipids from animal tissue. J Biol Chem. 1957;226:497-509.

21. Frisch RE, Hegsted DM, Yoshinaga K. Carcass components at first estrus of rats on high-fat and low-fat diets: Body water, protein, and fat. Proc Nat Acad Sci. 1977;74:379-83.
22. Brommage R. Validation and calibration of DEXA body composition in mice. Am J Physiol Endocrinol Metab. 2003;285:E454-9.

23. Fernández Vieitez JA. Validación por disección de cadáveres de 7 métodos antropométricos para estimar la masa muscular humana. Rev Cubana Aliment Nutr. 2001;15(2):115-20.

24. Rose BS, Flatt WP, Martin RJ, Lewis RD. Whole body composition of rats determined by dual energy $\mathrm{X}$-ray absorptiometry is correlated with chemical analysis. J Nutr. 1998;128:246-50.

25. Feely RS, Larkin LM, Halter JB, Dengel DR. Chemical versus dual energy $x$-ray absorptiometry for detecting age-associated body compositional changes in male rats. Exp Gerontol. 2000;35(3):417-27.

26. Hunter HL, Nagy TR. Body composition in a seasonal model of obesity: longitudinal measures and validation of DXA. Obes Res. 2002;10:1180-7.

27. Nagy TR, Clair A-L. Precision and accuracy of dual-energy $X$-ray absorptiometry for determining in vivo body composition of mice. Obes Res. 2000;8:392-8.

28. Punyanita M, Leibel RL, Heymsfeld SB, Boozer $\mathrm{CN}$. Evaluation of a new dual-energy X-ray absorptiometric technique for in vivo body composition measurements in mice (Abstract). FASEB J. 2000;14:A497.

Manuscrito recibido el 6 de abril de 2010 y aceptado para publicación el 10 de junio de 2010.

Correspondencia:

Dr. Marco Antonio Cossio Bolaños

Msc. Marco Antonio Cossio Bolaños

Av. Erico Verissimo 701.

Ciudad Universitaria CEP. 13083-851.

Campinas, S.P. Brasil.

E-mail: mcossio1972@hotmail.com 